\title{
NIVEL DE SUSTENTABILIDAD EN PEQUEÑAS EXPLOTACIONES SOJERAS DE LA PROVINCIA DEL CHACO
}

- SUELO AGRÍCOLA-

SUSANA G. DE GELMAN ROSA CRUZ DE INNOCENTE SUSANA ROSA JEREZ MARGARITA CARLOTA CARBAJAL IDALIA DE CASTRO

FACULTAD DE CIENCIAS ECONÓMICAS UNIVERSIDAD NACIONAL DEL NORDESTE

ARGENTINA

"Ser sustentable no es sólo lavar las culpas ni sólo cuidar el medio ambiente, sino ser socialmente justo, responsable con el ambiente y, por lo tanto, también económicamente viable". 


\title{
RESUMEN
}

n el Chaco se conjugan, la importancia económica del cultivo de soja cuyo avance
paulatino fue favorecido por múltiples factores externos e internos, con la signifi-
cación social de las pequeñas empresas agrícolas predominantemente familiares.
Sin embargo, la evaluación de su explotación sustentable avanzó a menor ritmo,
involucrando varias disciplinas con un desarrollo dispar. Por ello, es objetivo del presente tra-
bajo, describir las condiciones en las que se lleva a cabo la producción sojera y evaluar el grado
de sustentabilidad de las pequeñas empresas del Chaco en lo atinente a la protección del suelo.
Las decisiones metodológicas definieron un diseño no experimental, observacional, descrip-
tivo, transversal y prospectivo, cuya recolección de datos se implementó con un cuestionario
ad hoc. Los pequeños productores sojeros chaqueños fueron seleccionados como unidad de
análisis, en razón de su importancia económico-social y alta vulnerabilidad. La muestra, in-
tegrada por 27 participantes, predominantemente de sexo masculino (92.59\%) con una edad
promedio de 48.70 (DE = 12.91), resultaron escogidos en forma aleatoria de una población
seleccionada de manera intencional y accidental. Los resultados obtenidos, indican conduc-
tas permanentes que, en general, propenden a la protección de los recursos naturales. Entre
ellas: rotación de cultivos, siembra directa sobre rastrojos, semilla transgénica y monitoreo de
plagas bajo asistencia técnica; debiendo fortalecerse el cuidado preventivo del suelo mediante:
alternancia de actividades económicas, fertilización balanceada y estudios periódicos. Final-
mente, se anhela que éste trabajo, contribuya a una explotación sustentable y con la comuni-
dad científico-profesional involucrada.

Palabras clave: sustentabilidad, soja, agricultura familiar, pequeña empresa.

\begin{abstract}
Combine in the Chaco, the economic importance of soybean gradual progress which was favored by many external and internal factors, the social significance of small family predominantly agricultural enterprises. However, the assessment of their sustainable exploitation progressed at a slower pace, involving various disciplines with uneven development. Therefore, aim of this paper is to describe the conditions under which it conducts soybean production and assess the degree of sustainability of small businesses in the Chaco as it pertains to the protection of soil. Methodological decisions defined a non-experimental, observational, descriptive, cross-sectional and prospective data collection which im-mented with an ad hoc questionnaire. Chaco soy Smallholders were selected as the unit of analysis, because of their socio-economic importance and high vulnerability. The sample, consisting of 27 participants, predominantly male $(92.59 \%)$ with a mean age of 48.70 ( $\mathrm{SD}=12.91$ ), were chosen at random from a population selected intentionally and accidentally. The results obtained indicate that permanent conducts generally tend to protection of natural resources.
\end{abstract}


Among them: crop rotation, direct seeding into stubble, transgenic seed and pest monitoring under technical assistance strengthened preventive care must land by: alternation of economic activities, balanced fertilization and regular studies. Finally, longs for this work, contribute to a sustainable exploitation and professional scientific community involved.

Keywords: : sustainability, soy, family farming, small business.

\section{INTRODUCCIÓN}

En la Provincia del Chaco se conjugan, la importancia económica del cultivo de soja (cuyo avance paulatino se vio favorecido por múltiples factores), con la significación social de las pequeñas explotaciones agrícolas, predominantemente familiares.

Es así que, entre 1981 y 2011, la soja ha crecido en superficie implantada y cosechada, como en producción y rendimiento, a un ritmo promedio del 36\%, 38\%, $45 \%$ y $3 \%$ respectivamente. En la campaña 2010/2011, se implantaron 700.800 hectáreas, de las cuales se cosecharon 698.520, en las que se produjeron 1.655.117 toneladas a un rendimiento promedio de 2.369 kilogramos por hectárea cosechada. La campaña siguiente se perdió casi totalmente por la sequía.

Es el cultivo de mayor importancia relativa, así entre el 2005-2008, representó el 45\% de la producción agrícola de la provincia, ocupó una superficie promedio del 43,6\% y creció un $169 \%$ y $46 \%$ respectivamente, orientando el área agrícola disponible, ya sea con desplazamiento de cultivos tradicionales (especialmente algodón) o mediante la incorporación de nuevas tierras en un marcado avance sobre el monte nativo.

Sin embargo, la valoración integral de la actividad sojera, avanzó a un ritmo menor. La delimitación teórica del tema involucra: a) definiciones no unívocas de explotación sustentable originadas mayormente en las ciencias agrarias; $\mathrm{y}$ b) descripciones múltiples de la pequeña empresa, adoptándose las siguientes:

a) Explotación sustentable: es aquella que al desarrollar su actividad económica protege la integridad de los recursos naturales, es rentable y socialmente aceptada.

La integridad de los recursos naturales, está ligada a la implementación de "Buenas Prácticas Agrícolas” (Inciarte, 2004) consistentes en la aplicación del conocimiento disponible a la utilización sostenible de los recursos naturales básicos.

La rentabilidad se obtiene con una ecuación integral positiva de ingresos y costos. Los ingresos dependen del volumen de producción y del precio de venta fijado por el mercado, en tanto que cada elemento del costo tiene factores diferenciados que marcan su comportamiento e incidencia en la ecuación final.

La aceptación social, se vincula a la contribución que la explotación sojera genera para la comunidad en la que está inserta donde se vuelcan sus efectos positivos, vía pago de impuestos, sostenimiento del nivel de empleo y/o desarrollo de la actividad comercial, entre otros.

Las Buenas Prácticas Agrícolas -BPA-, constituyen un conjunto de herramientas cuyo 
uso persigue la sustentabilidad ambiental, económica y social, especialmente en pequeños productores subsistenciales. Son un componente de competitividad ya que se traducen en procesos de calidad relativamente nuevos ${ }^{1}$ en Argentina (Inciarte, 2004).

b) Pequeña empresa: en este estudio, está representada por el productor sojero del Chaco. Su descripción comparte características consideradas por Scheinkerman $(2007,2009)$ para "explotación agropecuaria familiar (EAP)" tales como: el trabajo directo y personal del agricultor, no más de dos trabajadores contratados y, determinados límites de extensión y superficie cultivada.

La importancia socio-económica de las pequeñas empresas agrícolas, pone de relieve analizar -a nivel microeconómico ${ }^{2}$ - en qué condiciones se debe llevar a cabo la producción sojera en las pequeñas empresas del Chaco para que se genere un efecto residual positivo; ya que si bien se pone en funcionamiento un enorme engranaje de servicios, insumos y transporte, es escasa la evaluación de su permanencia en condiciones sustentables. Ésta valoración resulta pertinente a las Ciencias Económicas, dado que, bajo una concepción sistémica, las decisiones que se adoptan en la empresa se traducen en cambios cualitativos o cuantitativos de su patrimonio que repercuten (en forma inmediata o diferida) en la rentabilidad, la que, conjuntamente con la conservación del suelo agrícola, condicionan inexorablemente la continuidad de la explotación.

Por otra parte, si bien los mayores avances de investigación refieren a la Región Pampeana (Scheinkerman, 2007, 2009; Casparri y García Fronti, 2011), dichos estudios no son directamente aprovechables para la Provincia del Chaco cuyo clima y suelo no presentan las mismas ventajas comparativas que la zona central del país. A modo de ejemplo, un pequeño productor es aquel que -entre otras características- tiene una explotación promedio de 1000 (Scheinkerman, 2007) o 162 hectáreas según se radique en la Región Pampeana o el Nordeste Argentino $^{3}$-NEA-, respectivamente. En este contexto, no se han hallado publicaciones referidas a la evaluación de la sustentabilidad de pequeñas empresas sojeras, menos aún para las establecidas en el Chaco.

Teniendo en cuenta todo lo mencionado en este punto, el interrogante formulado dio lugar a la siguiente hipótesis sustantiva: "la producción sojera genera efectos socio-económicos positivos, si las pequeñas empresas chaqueñas son explotaciones sustentables". Estrechamente asociado a la hipótesis que le da validez conceptual a este trabajo, el objetivo general consiste en describir las condiciones en las que se lleva a cabo la producción sojera y evaluar el grado de sustentabilidad de las pequeñas empresas chaqueñas en lo atinente a la protección del suelo.

\footnotetext{
${ }^{1}$ Antecedente normativo: Resolución 71/99 de la SAGPyA (Secretaría de Agricultura, Ganadería, Pesca y Alimentos) referida a hortalizas.

${ }^{2}$ Se estudian el comportamiento de las unidades económicas consideradas individualmente.

${ }^{3}$ Según los datos del Censo Nacional Agropecuario 2008, la Región Pampeana está conformada por las provincias de Buenos Aires, Entre Ríos, Santa Fe, Córdoba, La Pampa, y San Luis considerada la más importante del territorio argentino desde el punto de vista económico, ya que sus características de clima y suelo la transforman en una zona agrícola y ganadera por excelencia. La Región NEA se integra por las provincias del Chaco, Corrientes, Formosa y Misiones.
} 


\section{MATERIALES Y MÉTODO}

Se ha estimado conveniente que este trabajo se desarrolle siguiendo la estructura clásica de las investigaciones que se realizan en esta línea. Se divide en dos partes, la primera corresponde al análisis teórico y la segunda se relaciona con el estudio empírico, en cuyo marco se plantean la discusión de los resultados y las conclusiones.

Para establecer las características metodológicas asumidas, se indican en el presente apartado: el diseño de la investigación, los participantes, las variables consideradas en el estudio, el instrumento de medición y el procedimiento utilizado para recoger los datos.

\subsection{Diseño de la investigación}

Difícilmente el diseño de una investigación se apoye sólo en métodos puros, más aún cuando los criterios (o sus categorías) de selección no son mutuamente excluyentes. Por lo tanto, es común que se presenten características de un conjunto variado de ambos elementos.

En vista de lo señalado, esta investigación, en función del objetivo que busca es inicialmente de naturaleza no experimental y observacional; en un segundo momento explicativa. Si se considera como criterio el tipo de información que interesa y el modo de recogerla, es de estilo descriptivo y; en atención a la forma de administrar el instrumento de medición, se empleó la técnica del cuestionario.

A su vez, si se tiene en cuenta el marco donde se llevó a cabo, es una investigación de campo. Asimismo en razón de cómo se miden, analizan y recolectan los datos, este estudio es de línea cuanti-cualitativa, de corte transversal y de tipo prospectivo ya que su propósito es diagnosticar y plantear alternativas para el desarrollo de la actividad productiva, económica y financiera.

\subsection{Participantes}

Universo: la revisión bibliográfica previa aportó las definiciones enunciadas de: explotación sustentable y pequeña empresa mencionadas en el primer apartado. En tanto que, la imposibilidad fáctica de aplicar al NEA los resultados de estudios realizados en la Región Pampeana, obligó a adecuar la descripción de Scheinkerman $(2007,2009)$ para dar lugar al Pequeño productor sojero chaqueño.

El universo ideal ${ }^{4}$ de esta investigación se configura por aquellos que cumplen con todas las características cuantitativas y cualitativas que a continuación se enuncian:

a) La explotación no supera: quinientas (500) hectáreas de superficie total, doscientas (200) hectáreas de superficie cultivada y, dos (2) empleados no familiares remunerados.

b) Los productores: son personas físicas que trabajan directamente en su explotación agrícola (empresas unipersonales).

Población: Se estimó pertinente centralizar la investigación empírica, en el espacio geográfico 
de la subzona XVI-B5 (INTA, 2010), la que, consistente con campañas anteriores, concentra el $55 \%$ (349.500 ha) de la superficie total (639.00o ha) cultivada con soja en el Chaco (INTA, 2011).

El equipo de investigación definió el criterio para seleccionar los sujetos que podían facilitar información. Por consiguiente, la selección de la población (en cuanto se refiere al tamaño de empresa y zona geográfica) fue de carácter intencional, dado que incluyó a ochenta y cuatro productores sojeros con explotaciones de hasta 200 hectáreas cultivadas, ubicadas en la sub zona XVI-B, que configuraron la población de este estudio.

Muestra: Para la aplicación del instrumento ad hoc, se efectuó una selección probabilística del grupo definitivo. Así, la muestra aceptante (Fox, 1981) de pequeños productores sojeros chaqueños quedó conformada por individuos escogidos utilizando el método aleatorio simple, teniendo todos ellos la misma probabilidad de integrarla.

La representatividad se encuentra respaldada por las siguientes características:

a) Contiene todos los departamentos de la zona agrícola que aportó la mayor parte de la superficie sembrada con soja en el Chaco durante el año 2011 y anteriores.

b) La ubicación geográfica de las empresas se realizó a partir de estudios previos realizados por el Instituto Nacional de Tecnología Agropecuaria (INTA).

c) El tamaño muestral es equivalente al $32.14 \%$ de la población, por lo que se presume que el número de observaciones realizadas es razonable.

d) El método probabilístico de extracción de los casos particulares que fueron entrevistados, consolidaron las fortalezas de la representatividad.

Conforme lo expresado sobre población y muestra, y a efectos de sustentar las decisiones metodológicas asumidas se sostiene que:

1) El muestreo no probabilístico para determinar la población, significó la adopción de un criterio subjetivo e implicó: a) un esfuerzo deliberado para obtener una muestra "representativa" mediante la inclusión de sujetos típicos; y b) la optimización de los recursos disponibles. Sin embargo, no resultó arbitrario, sino de la conjunción de antecedentes relevantes y pertinentes, lo que acotó las limitaciones emergentes de este tipo de muestreo.

2) El muestreo probabilístico utilizado en la extracción de la muestra aceptante, concede mayor rigurosidad metodológica y representatividad. Así, las conclusiones obtenidas explican a toda la población aunque no permitirían inferir sobre el universo.

Unidades de análisis: La elección de los pequeños productores sojeros chaqueños como unidad de análisis se efectuó por: su importancia económico-social y su vulnerabilidad.

\footnotetext{
${ }^{5}$ La Provincia del Chaco está segmentada en cuatro zonas agropecuarias identificadas como XVI: Centro oeste del Chaco, XVII: Ganadera del este, XVIII: Mixta del norte y XIX: Noroeste del Chaco; siendo de particular interés, por su fuerte concentración agrícola, la zona XVI que se divide en tres sub zonas:

* XVI-A: Central del Chaco: Departamentos Comandante Fernández, Independencia, Quitilipi y Maipú.

* XVI-B: del Oeste del Chaco con epicentro en la localidad de General Pinedo e integrada por los Departamentos: General Belgrano, 9 de Julio, Chacabuco, 12 de Octubre y 2 de Abril.

* XVI-C: Mixta Sudoeste del Chaco: Departamentos de O’Higgins, San Lorenzo, Fontana, y Santa María de Oro.
} 
Fuente de datos: La escasa capacidad para actuar sobre (o transformar) el objeto de investigación, como así también la posibilidad real y valiosa de acceder al productor en forma directa, determinaron una fuente de datos testimonial, proveniente exclusivamente de manifestaciones ${ }^{6}$ del agricultor ante las distintas preguntas y aclaraciones efectuadas, utilizando como técnica la encuesta y como instrumento de observación un cuestionario-entrevista descripto en el punto 2.4 .

\subsection{Variables consideradas en el estudio}

Las variables teóricas se desprenden de la definición de explotación sustentable (protección de los recursos naturales, rentabilidad y aceptación social). Asimismo, la protección del recurso natural suelo se corresponde con las variables empíricas: modelo de producción utilizado, técnicas agrícolas empleadas, uso de agroquímicos y disponibilidad de asistencia técnica. Los resultados obtenidos en el análisis de los datos recolectados se indican en el tercer apartado de este trabajo.

\subsection{Instrumento de medición}

Con el propósito de recoger los datos relativos al tema bajo estudio se diseño ad hoc un instrumento denominado "Cuestionario de Sustentabilidad en Pequeñas Explotaciones Agrícolas Sojeras de la Provincia del Chaco (PEASPC)” el que obra en Anexo I. El mismo consta de cuatro bloques en total, con las siguientes características: el primero sirve para identificar a los productores, el segundo tiene catorce preguntas de tipo cerradas que se responden calificando sobre la base de una escala tipo Likert de 5 puntos $^{7}$. El tercer grupo de preguntas dispone de columnas donde se registran datos numéricos de tres campañas agrícolas y como mínimo se anotan los que corresponden a la última. En el cuarto y último bloque, se encuentran cinco ítems, que de corresponder, se solicita que comente detalles de ciertas decisiones adoptadas.

El instrumento de medición ha sido validado cualitativamente mediante los métodos: a) juicio de expertos y b) grado de acuerdo. El primero, proporciona indicadores subjetivos de validez y el segundo aporta lo que algunos autores llaman indicadores de la validez factorial. La evaluación es necesaria y relevante pues indica en que grado la prueba será capaz de medir lo que realmente pretende, cuyas condiciones de aplicación se describen en el próximo apartado.

\subsection{Procedimiento}

La etapa de recolección de datos, instancia delicada en toda investigación de línea empírica, debe ser cuidadosamente planificada a efectos de evitar las dificultades habituales que se presentan al implementarla y que, de hecho, han surgido. En virtud de ello, se expone un resumen del procedimiento aplicado en el trabajo de campo. 
En primer lugar fue importante la colaboración de delegados de la Agencia de Desarrollo Rural del Ministerio de Producción del Gobierno de la Provincia, quienes aportaron datos para la ubicación geográfica de las explotaciones sojeras.

La recolección de datos se llevó a cabo durante septiembre de 2012, mediante entrevistas cuya duración promedio fue de treinta minutos, realizadas en la residencia de los productores, tarea que estuvo a cargo de tres docentes-investigadores, previa consulta con el productor, a fin de acordar el horario de los encuentros.

En aquel primer contacto, se les informó que su colaboración voluntaria posibilitaría la realización de una investigación desarrollada en el marco de proyecto acreditado por la Universidad Nacional del Nordeste; y que los datos recogidos serían manejados con carácter estrictamente confidencial y utilizados solamente con fines científicos. Se les indicó también, la importancia de responder sinceramente.

Concluido el trabajo de campo propiamente dicho y el ordenamiento de la información obtenida, se procedió a la construcción de la base de datos en formato electrónico.

\section{RESULTADOS}

Una de las bondades que caracteriza a las investigaciones empíricas es que, a través de esta línea de estudio, es posible contrastar el concepto teórico que se sostiene con los datos de la muestra; es decir, brinda la oportunidad de evaluar en la realidad el grado de ajuste entre el paradigma que se hipotetiza y los resultados que se obtienen.

Por ello, se ha realizado un examen estadístico descriptivo de los datos con la finalidad de: a) explorar el comportamiento de las variables del cuestionario ad hoc con el propósito final de obtener información sobre las particularidades que en la realidad presenta el tema objeto de interés; $\mathrm{y}$ b) comprobar en qué medida los resultados alcanzados están en consonancia con los proporcionados por la literatura especializada.

Este proceso se ha llevado a cabo luego de realizar el control de la matriz de datos -en formato electrónico-, y con asistencia del programa informático SPSS 15.0., lo que permitió determinar los estadísticos indicados en la Tabla 1.

Tabla 1. Análisis estadísticos implementados en esta investigación.

\begin{tabular}{|l|l|l|}
\hline Dominio & Estudios estadísticos & Indicadores \\
\hline \multirow{3}{*}{ Descriptivo } & * De tendencia central & Media, moda y mediana. \\
& * De distribución & Desviación estándar, mínimo, máximo y rango. \\
& * Dablas de frecuencias, asimetría y curtosis. \\
\hline
\end{tabular}

Asimismo, dado el número de variables que se estudian, son numerosos los análisis estadísticos que podrían tener lugar en este apartado. Sin embargo, como es evidente, las pretensiones de 
este estudio van más allá de limitarse a brindar meros valores, por esta razón sólo se presentarán seguidamente, de forma sintética, aquellos indicadores que se consideran más adecuados para caracterizar la muestra en las diferentes variables a nivel de centralización, dispersión y distribución.

\subsection{Resultados obtenidos relativos a datos personales}

En primer término se presenta la información relacionada con datos personales (edad y género) de los participantes en el estudio y se analiza la ubicación de sus explotaciones agrícolas.

Para los datos de la muestra, en la Tabla 2 pueden observarse algunos indicadores correspondientes a la variable edad. Ante la existencia de múltiples modas y con la intención de que ésta, junto con los estadísticos media y mediana, refleje que los datos se asemejan a una distribución normal, se adoptó aquel valor modal más cercano a los otros dos índices nombrados.

Tabla 2. Estadísticos descriptivos de la variable "edad"

\begin{tabular}{|c|c|c|c|c|c|c|c|c|c|}
\hline Muestra & \multicolumn{3}{|c|}{ Centralización } & \multicolumn{4}{|c|}{ Dispersión } & \multicolumn{2}{|c|}{ Distribución } \\
\hline $\mathrm{N}$ & Media & Mediana & Moda & $\mathrm{DE}$ & Rango & Mínimo & Máximo & Asimetría & Curtosis \\
\hline 27 & 48.70 & 45.00 & 40.00(a) & 12.91 & 55.00 & 30.00 & 85.00 & 0.90 & 0.70 \\
\hline
\end{tabular}

(a) Existen múltiples modas, siendo indicada la menor de ellas.

Fuente: Elaboración propia

Según se desprende de la Tabla 2, los productores que colaboraron en la etapa empírica de la investigación, poseen edades que oscilan entre 30 y 85 años, con un promedio de 48.70 años y desviación estándar de 12.91. En la Tabla 3, se muestran las frecuencias de la variable edad, en intervalos de cinco años que agrupan las observaciones pertinentes a cada franja etarea. Con el fin de presentar gráficamente lo expresado, se muestra también, el histograma con curva normal en Figura 1, correspondiente a los valores observados.

Tabla 3. Edad de los productores

\begin{tabular}{|c|c|c|c|}
\hline Años & Frecuencia & Porcentaje & $\begin{array}{c}\text { Porc. } \\
\text { Acumulado }\end{array}$ \\
\hline $27.5-32.5$ & 1 & 3.70 & 3.70 \\
\hline $32.5-37.5$ & 4 & 14.82 & 18.52 \\
\hline $37.5-42.5$ & 6 & 22.22 & 40.74 \\
\hline $42.5-47.5$ & 4 & 14.82 & 55.56 \\
\hline $47.5-52.5$ & 2 & 7.41 & 62.97 \\
\hline $52.5-57.5$ & 3 & 11.11 & 74.08 \\
\hline $57.5-62.5$ & 4 & 14.82 & 88.90 \\
\hline $62.5-67.5$ & 1 & 3.70 & 92.60 \\
\hline $67.5-72.5$ & 1 & 3.70 & 96.30 \\
\hline $72.5-77.5$ & 0 & 0.0 & 96.30 \\
\hline $77.5-82.5$ & 0 & 0.0 & 96.30 \\
\hline $82.5-87.5$ & 1 & 3.70 & 100.00 \\
\hline Total & 27 & 100.00 & \\
\hline
\end{tabular}

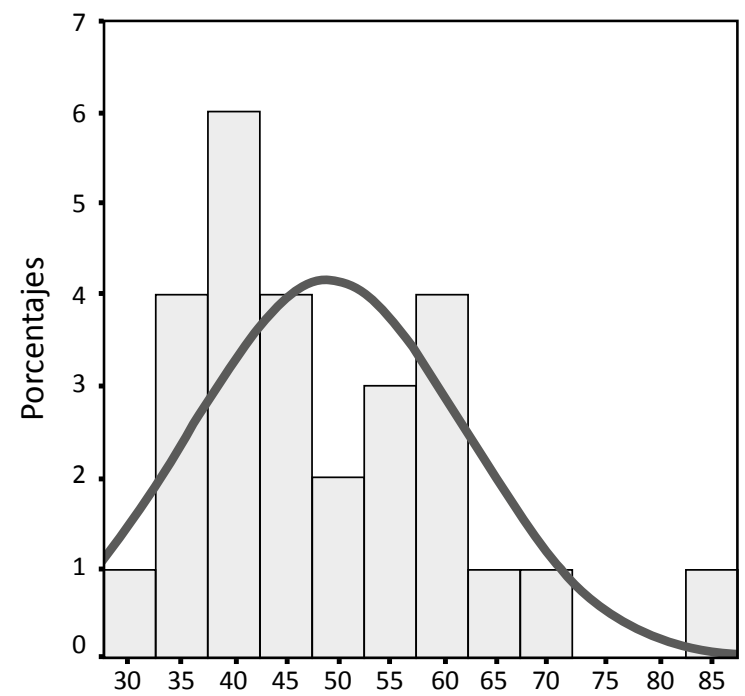

Figura 1. Histograma con curva normal de los valores observados en la variable "edad". 
La altura de las barras del histograma representa el número de productores comprendidos en cada una de ellas (la suma de los valores es 27), mientras que el ancho indica el intervalo de edades en el que se ubican. Así, por ejemplo, la segunda barra del histograma, refiriéndonos de izquierda a derecha, está conformada por cuatro (4) productores, tres (3) de los cuales tienen 35 años y uno (1) de ellos 37 años.

La distribución de los datos es claramente asimétrica positiva (As $=0.90$ ) o sesgada a la derecha (el valor resultante según Tabla 2 fue superior a 0.5 , que es el nivel de tolerancia aconsejado para aceptar la simetría de la distribución), dado que la media (48.70) ha resultado mayor que la mediana. En este contexto significa que los productores menores de 45.00 años (mediana) están atomizados en un rango de quince (15) años que comienza a la edad de 30.oo, en tanto los que superan la mediana se dispersan en un rango de cuarenta (40) años llegando hasta los 85.00, por lo que la cola derecha de la curva es visiblemente más larga que la izquierda (véase Figura 1).

Por su parte, el coeficiente de curtosis $(C u r t=0.70)$ indica un elevado grado de concentración de los valores en la región central de la distribución (excede el nivel 0.5 de tolerancia aconsejado para aceptar que los datos se encuentran normalmente distribuidos), lo que es debido a que 23 de los 27 productores que participaron se encuentran entre la media más/menos una desviación estándar, concentrando en ese intervalo de edades el 85.18\% de los datos ${ }^{8}$. En definitiva, se trata de una curva que comparada con la normal resulta más puntiaguda, conocida debido a esta característica con el nombre leptocúrtica.

En cuanto al género de los 27 productores entrevistados, 25 de ellos; esto es, el 92.59\%, corresponde al sexo masculino, mientras que los 2 restantes son de género femenino; es decir, el 7.41\%.

Para analizar la dispersión geográfica de las empresas, se debe tener en cuenta que algunos de los veintisiete integrantes de la muestra poseen más de un establecimiento (campo), dando lugar a veintinueve observaciones respecto de la variable localización del emprendimiento. Como surge de la Tabla 4, se destacan el departamento Chacabuco y 12 de Octubre, con el $51.72 \%$ y el 31.03\% respectivamente del total de los datos muestrales. Ambos, cuyas ciudades de cabecera son Charata y General Pinedo, separadas a una distancia de quince kilómetros aproximadamente, conforman el núcleo del polo agrícola sojero de la Provincia del Chaco.

Tabla 4. Localización del emprendimiento

\begin{tabular}{|c|c|c|c|c|c|c|c|}
\hline Localización & $\begin{array}{c}\text { General } \\
\text { Belgrano }\end{array}$ & $\begin{array}{c}\mathbf{9} \text { de } \\
\text { Julio }\end{array}$ & Chacabuco & $\begin{array}{c}\mathbf{1 2} \text { de } \\
\text { Octubre }\end{array}$ & $\begin{array}{c}\mathbf{2} \text { de } \\
\text { Abril }\end{array}$ & $\begin{array}{c}\text { Otros } \\
\text { Dptos. }\end{array}$ & Total \\
\hline Establecimientos & 0.00 & 3 & 15 & 9 & 0.00 & 2 & 29 \\
\hline Porcentaje & $0.00 \%$ & $10.35 \%$ & $51.72 \%$ & $31.03 \%$ & $0.00 \%$ & $6.90 \%$ & $100.00 \%$ \\
\hline
\end{tabular}

Fuente: Elaboración propia 
Las decisiones del productor vinculadas con la protección del recurso natural suelo, deberían orientarse a la observancia de buenas prácticas agrícolas entre las cuales se recomiendan: 1) no remoción, presencia de cobertura; 2) rotación de cultivos; 3) manejo integrado de malezas, enfermedades, insectos y otras plagas; 4) manejo eficiente y responsable de agroquímicos; 5) nutrición estratégica (Aapresid, s/f).

Es así que el cuestionario utilizado -obrante en Anexo I-, contiene preguntas que identifican atributos o cualidades del modelo productivo utilizado, las técnicas agrícolas implementadas, el uso de agroquímicos y el empleo de asistencia técnica, los que determinan el grado en que la empresa protege el suelo agrícola. Respecto de las variables analizadas en este trabajo (preguntas 1 a 12) se destaca la moda como única medida de tendencia central y resultan pertinentes las tablas de frecuencia.

3.2.1. Modelo de producción (Ítems 1,2 y 3): involucra el estudio de las decisiones adoptadas que propician la biodiversidad, relacionadas con: la rotación ${ }^{9}$ entre el cultivo de soja y otros compatibles con él (sorgo, maíz, trigo, entre otros). En el mediano plazo, la alternancia de actividades agricultura/ganadería perfecciona el modelo, propendiendo al desarrollo sustentable de la actividad. El relevamiento se muestra en la Tabla 5.

Tabla 5: Modelo de producción

\begin{tabular}{|l|c|c|c|c|c|c|}
\hline \multirow{3}{*}{ Opciones } & \multicolumn{5}{|c|}{ Adopción de prácticas recomendadas } \\
\cline { 2 - 7 } & \multicolumn{3}{|c|}{$\begin{array}{c}\text { Rotación de soja } \\
\text { con otros cultivos }\end{array}$} & \multicolumn{2}{c|}{$\begin{array}{c}\text { Alternancia de agricultura } \\
\text { con ganadería }\end{array}$} \\
\cline { 2 - 7 } & $\begin{array}{c}\text { Frecuen- } \\
\text { cia }\end{array}$ & $\begin{array}{c}\text { Porcen- } \\
\text { taje }\end{array}$ & $\begin{array}{c}\text { Porc. } \\
\text { acum. }\end{array}$ & $\begin{array}{c}\text { Frecuen- } \\
\text { cia }\end{array}$ & $\begin{array}{c}\text { Porcen- } \\
\text { taje }\end{array}$ & $\begin{array}{c}\text { Porc. } \\
\text { acum. }\end{array}$ \\
\hline Desconoce & 1 & 3.70 & 3.70 & 1 & 3.70 & 3.70 \\
\hline Nunca & 1 & 3.70 & 7.40 & 19 & 70.37 & 74.07 \\
\hline Pocas veces & 6 & 22.22 & 29.62 & 4 & 14.82 & 88.89 \\
\hline Muchas veces & 1 & 3.70 & 33.32 & 1 & 3.70 & 92.59 \\
\hline Permanentemente & 18 & 66.68 & 100.00 & 2 & 7.41 & 100.00 \\
\hline Total & 27 & 100.00 & & 27 & 100.00 & \\
\hline
\end{tabular}

Fuente: Elaboración propia

Las conclusiones más relevantes que surgen de la Tabla 5 son: $a$ ) del total de productores encuestados el 70.38\% (3.70\% muchas veces o $66.68 \%$ permanentemente) rotan sus cultivos con distinta intensidad y; $b$ ) resultan escasas las observaciones sobre alternancia de actividades, la que alcanza un valor del $11.11 \%$ (3.70\% muchas veces o 7.41\% permanentemente). 
3.2.2. Técnicas agrícolas (Ítems 4 y 5): Las dimensiones que informan sobre este aspecto son: la siembra directa ${ }^{10}$, implantación sobre rastrojos y la utilización de semilla transgénica ${ }^{11}$, los datos se transcriben en la Tabla 6.

Tabla 6: Técnicas agrícolas

\begin{tabular}{|l|c|c|c|c|c|c|c|c|c|}
\hline \multirow{3}{*}{ Opciones } & \multicolumn{8}{|c|}{ Adopción de prácticas recomendadas } \\
\cline { 2 - 13 } & \multicolumn{2}{|c|}{ Siembra Directa } & \multicolumn{2}{|c|}{$\begin{array}{c}\text { mplantación sobre ras- } \\
\text { trojos del cultivo anterior }\end{array}$} & \multicolumn{2}{c|}{ Semilla transgénica } \\
\cline { 2 - 12 } & $\begin{array}{c}\text { Frecuen- } \\
\text { cia }\end{array}$ & $\begin{array}{c}\text { Porcen- } \\
\text { taje }\end{array}$ & $\begin{array}{c}\text { Porc. } \\
\text { acum. }\end{array}$ & $\begin{array}{c}\text { Frecuen- } \\
\text { cia }\end{array}$ & $\begin{array}{c}\text { Porcen- } \\
\text { taje }\end{array}$ & $\begin{array}{c}\text { Porc. } \\
\text { acum. }\end{array}$ & $\begin{array}{c}\text { Frecuen- } \\
\text { cia }\end{array}$ & $\begin{array}{c}\text { Porcen- } \\
\text { taje }\end{array}$ & $\begin{array}{c}\text { Porc. } \\
\text { acum. }\end{array}$ \\
\hline Nunca & 2 & 7.41 & 7.41 & 8 & 29.63 & 29.63 & 1 & 3.70 & 3.70 \\
\hline Pocas veces & 4 & 14.82 & 22.23 & 8 & 29.63 & 59.26 & 4 & 14.82 & 14.82 \\
\hline Muchas veces & 1 & 3.70 & 25.93 & 2 & 7.41 & 66.67 & 2 & 7.41 & 7.41 \\
\hline Permanentemente & 20 & 74.07 & 100.00 & 9 & 33.33 & 100.00 & 20 & 74.07 & 74.07 \\
\hline Total & 27 & 100.00 & & 27 & 100.00 & & 27 & 100.00 & 100.00 \\
\hline
\end{tabular}

Fuente: Elaboración propia

Puede observarse en la Tabla 6, que 20 de los 27 entrevistados emplea permanentemente la siembra directa y semillas con distinto grado de modificación genética (transgénica), lo que representa el 74.07\%, en tanto que sólo el 40.74\% de ellos siembra (7.41\% muchas veces o $33.33 \%$ permanentemente) sobre rastrojos del cultivo inmediato anterior. Estas observaciones son indicativas de decisiones que contribuyen a la sustentabilidad ambiental de la explotación, toda vez que: a) la labranza convencional provoca distintos niveles de deterioro dado que al remover la tierra, incorpora oxígeno generando combustión lo que destruye su materia orgánica; y b) el uso de semillas no transgénicas requiere una mayor aplicación de agroquímicos.

3.2.3. Uso de agroquímicos (Ítems 6 a 8): El conocimiento profundo del cultivo, implica potenciar el uso de los recursos para incrementar rendimientos, eligiendo los materiales genéticos adecuados (semilla), fechas de siembra, cultivos antecesores (prácticas de rotación), densidad (plantas por hectárea) y estructura del cultivo (distancia de siembra), por mencionar algunos factores que permitirán atenuar el empleo de agroquímicos que impacten al medio ambiente (Carbajal, Innocente, Jerez, de Castro y García; 2011).

\footnotetext{
${ }^{10}$ La siembra directa (o labranza cero) es una técnica por medio de la cual se deposita simultáneamente la semilla y el fertilizante en una angosta ranura oradada por el abresurcos de la sembradora, sin realizar movimientos importantes de suelo (ni araduras ni rastrajes). Puede ser optimizada si se la efectúa sobre rastrojos del cultivo anterior, dejando sobre la superficie del suelo una capa de biomasa aérea residual del cultivo anterior que aporte materia orgánica, con el objetivo de remover lo menos posible el suelo, disminuir los ciclos de oxigenación intensos de la materia orgánica evitando la destrucción de la misma.

${ }^{11}$ Semillas de soja genéticamente modificadas que son resistentes al glifosato (herbicida no selectivo de amplio espectro, desarrollado para eliminación de hierbas y de arbustos, en especial los perennes, que es absorbido por las hojas y no por las raíces).
} 
Consecuentemente, el manejo integrado de plagas requiere el monitoreo ${ }^{12}$ constante de las amenazas al cultivo y si es necesario se procede al control ${ }^{13}$ de malezas y plagas. El esquema se completa con las decisiones referidas la fertilización del suelo. Los datos sobre este tópico se evidencian en la Tabla 7.

Tabla 7: Uso de agroquímicos

\begin{tabular}{|l|c|c|c|c|c|c|c|c|c|}
\hline \multirow{3}{*}{ Opciones } & \multicolumn{8}{|c|}{ Adopción de prácticas recomendadas } \\
\cline { 2 - 12 } & \multicolumn{2}{|c|}{ Monitoreo de plagas } & \multicolumn{3}{c|}{$\begin{array}{c}\text { Control de } \\
\text { plagas invasoras }\end{array}$} & \multicolumn{3}{c|}{$\begin{array}{c}\text { Fertilización } \\
\text { balanceada }\end{array}$} \\
\cline { 2 - 12 } & $\begin{array}{c}\text { Frecuen- } \\
\text { cia }\end{array}$ & $\begin{array}{c}\text { Porcen- } \\
\text { taje }\end{array}$ & $\begin{array}{c}\text { Porc. } \\
\text { acum. }\end{array}$ & $\begin{array}{c}\text { Frecuen- } \\
\text { cia }\end{array}$ & $\begin{array}{c}\text { Porcen- } \\
\text { taje }\end{array}$ & $\begin{array}{c}\text { Porc. } \\
\text { acum. }\end{array}$ & $\begin{array}{c}\text { Frecuen- } \\
\text { cia }\end{array}$ & $\begin{array}{c}\text { Porcen- } \\
\text { taje }\end{array}$ & $\begin{array}{c}\text { Porc. } \\
\text { acum. }\end{array}$ \\
\hline Nunca & 1 & 3.70 & 3.70 & 2 & 7.41 & 7.41 & 16 & 59.26 & 59.26 \\
\hline Pocas veces & 2 & 7.41 & 11.11 & 1 & 3.70 & 11.11 & 6 & 22.22 & 81.48 \\
\hline Muchas veces & 2 & 7.41 & 18.52 & 2 & 7.41 & 18.52 & 3 & 11.11 & 92.59 \\
\hline Permanentemente & 22 & 81.48 & 100.00 & 22 & 81.48 & 100.00 & 2 & 7.41 & 100.00 \\
\hline Total & 27 & 100.00 & & 27 & 100.0 & & 27 & 100.00 & \\
\hline
\end{tabular}

Fuente: Elaboración propia

Puede apreciarse en Tabla 7 que: a) el $81.48 \%$ de los productores vigilan permanentemente los agentes invasores del cultivo y debe reducirlos mediante la aplicación de distintos plaguicidas (insecticidas y/o herbicidas); y b) sólo el 18.52\% (11.11\% muchas veces o 7.41\% permanentemente) de ellos incorporan nutrientes específicos (azufre, fósforo, potasio, nitrógeno, calcio, magnesio, entre otros) al suelo que permitan el mejoramiento, mantenimiento y/o recuperación de los componentes consumidos durante cada campaña agrícola, evitando suelos inducidos por un desequilibrio en el potencial ${ }^{14}$ de hidrógeno "ph".

3.2.4. Empleo de asistencia técnica (Ítem 9 al 12): La empresa agrícola requiere asistencia técnica en distintas áreas de su funcionamiento; sin embargo, es difícil soslayar la importancia del asesoramiento agronómico. Como se puede apreciar en la Tabla 8, el 29.63\% (3.70\% muchas veces o $25.93 \%$ permanentemente) de los productores contrata asesoramiento especializado, en tanto que el $66.66 \%$ (7.41\% muchas veces o 59.25\% permanentemente) lo recibe de terceros (proveedores de insumos y entes gubernamentales). De lo anterior se infiere que el $96.29 \%$ (29.63\% contrata o $66.66 \%$ recibe) de la muestra cuenta con transferencia tecnológica.

\footnotetext{
${ }^{12}$ Consiste en la identificación y recuento de las plagas (malezas e insectos) que están afectando el cultivo y su evolución respecto del "umbral de daños", entendiendo por umbral de daños la "cantidad de habitantes de la población a partir de la cual es necesaria la aplicación de agroquímicos que tiendan a su destrucción". Por debajo de esa población no es necesaria ninguna acción directa pues no representa un riesgo para el cultivo y además el ecosistema los combate.

${ }^{13}$ Destrucción de plagas que exceden el umbral de daños, mediante la utilización de agroquímicos selectivos (de acuerdo al agresor que afecta el cultivo) o totales, siendo recomendables los primeros.

${ }^{14} \mathrm{El}$ potencial de hidrogeno muestra el grado de alcalinidad o acidez del suelo, factor responsable en la mayor o menor asimilabilidad de los nutrientes. El nivel normal es 7, por debajo de ese valor son suelos ácidos y por encima de él alcalinos.

${ }^{15}$ Surge de la consideración de dos preguntas no excluyentes, consecuentemente, puede ocurrir que un mismo productor contrate y reciba asesoramiento. En ese caso, el porcentaje estaría sobre estimado.
} 
Tabla 8: Disponibilidad de asistencia técnica

\begin{tabular}{|l|c|c|c|c|c|c|}
\hline \multirow{2}{*}{ Opciones } & \multicolumn{3}{|c|}{$\begin{array}{c}\text { Contrata asesoramiento } \\
\text { técnico }\end{array}$} & \multicolumn{3}{c|}{$\begin{array}{c}\text { Recibe asesoramiento } \\
\text { técnico de terceros }\end{array}$} \\
\cline { 2 - 7 } & $\begin{array}{c}\text { Frecuen- } \\
\text { cia }\end{array}$ & $\begin{array}{c}\text { Porcen- } \\
\text { taje }\end{array}$ & $\begin{array}{c}\text { Porc. } \\
\text { acum. }\end{array}$ & $\begin{array}{c}\text { Frecuen- } \\
\text { cia }\end{array}$ & $\begin{array}{c}\text { Porcen- } \\
\text { taje }\end{array}$ & $\begin{array}{c}\text { Porc. } \\
\text { acum. }\end{array}$ \\
\hline Desconoce & 2 & 7.41 & 7.41 & 1 & 3.70 & 3.70 \\
\hline Nunca & 14 & 51.85 & 59.26 & 4 & 14.82 & 18.52 \\
\hline Pocas veces & 3 & 11.11 & 70.37 & 4 & 14.82 & 33.34 \\
\hline Muchas veces & 1 & 3.70 & 74.07 & 2 & 7.41 & 40.75 \\
\hline Permanentemente & 7 & 25.93 & 100.00 & 16 & 59.25 & 100.00 \\
\hline Total & 27 & 100.00 & & 27 & 100.00 & \\
\hline
\end{tabular}

Fuente: Elaboración propia

En cuanto a evaluación cualitativa de las propiedades del suelo, en la Tabla 9 se advierte que un $11.11 \%$ de los entrevistados manifestó realizar periódicamente estudios de suelo ${ }^{16}(3.70 \%$ muchas veces o $7.41 \%$ permanentemente) y $14.82 \%$ (7.41\% muchas veces o $7.41 \%$ permanentemente) realiza el balance de nutrientes en forma regular. Siendo deseable que una mayor cantidad de productores adopten conductas de esta índole, en pos del uso sustentable del recurso.

Tabla 9: Propiedades del suelo

\begin{tabular}{|l|c|c|c|c|c|c|}
\hline \multirow{3}{*}{ Opciones } & \multicolumn{6}{|c|}{ Decisiones } \\
\cline { 2 - 8 } & \multicolumn{3}{|c|}{ Estudio de suelo } & \multicolumn{2}{c|}{ Balance de nutrientes } \\
\cline { 2 - 7 } & $\begin{array}{c}\text { Frecuen- } \\
\text { cia }\end{array}$ & $\begin{array}{c}\text { Porcen- } \\
\text { taje }\end{array}$ & $\begin{array}{c}\text { Porc. } \\
\text { acum. }\end{array}$ & $\begin{array}{c}\text { Frecuen- } \\
\text { cia }\end{array}$ & $\begin{array}{c}\text { Porcen- } \\
\text { taje }\end{array}$ & $\begin{array}{c}\text { Porc. } \\
\text { acum. }\end{array}$ \\
\hline Desconoce & 3 & 11.11 & 11.11 & 2 & 7.41 & 7.41 \\
\hline Nunca & 16 & 59.26 & 70.37 & 21 & 77.77 & 85.18 \\
\hline Pocas veces & 5 & 18.52 & 88.89 & 0 & 0.0 & 85.18 \\
\hline Muchas veces & 1 & 3.70 & 92.59 & 2 & 7.41 & 92.59 \\
\hline Permanentemente & 2 & 7.41 & 100.00 & 2 & 7.41 & 100.00 \\
\hline Total & 27 & 100.00 & & 27 & 100.00 & \\
\hline
\end{tabular}

Fuente: Elaboración propia

Finalmente, es importante recordar que el cuestionario utilizado en la recolección de los datos analizados, posee la capacidad adecuada para evaluar el concepto que se pretendía medir; es decir, reafirma la validez cualitativa, tanto del constructo, como de su contenido, la cual ha sido contrastada por especialistas en el área de conocimiento a través de los métodos: a) juicio 
de expertos, y b) grado de acuerdo. De hecho, el relevamiento se llevó a cabo con absoluta normalidad, no habiéndose recibido ningún tipo de sugerencias o expresiones relevantes, por parte de los productores encuestados.

Dicho instrumento fue diseñado para la observación sistemática de las tres variables teóricas consideradas en la definición de explotación sustentable, y por lo tanto, ha proporcionado datos muestrales de cada una de ellas. Sin embargo este estudio se concentra en los referidos únicamente a la protección del suelo, encontrándose en procesamiento y análisis los pertinentes a rentabilidad y aceptación social.

\section{DISCUSIÓN}

Los resultados obtenidos a partir de los análisis de datos efectuados en el punto anterior permiten llevar a cabo la correspondiente discusión acerca de los mismos. El objeto de este proceso previo es que sirva de punto de partida para presentar a continuación, a través de las conclusiones, los aportes más relevantes que este estudio anhela realizar a la comunidad científica y profesional. En ese sentido, se presentan: a) algunas apreciaciones acerca de las estimaciones realizadas, b) el planteo de investigaciones complementarias, y c) las propuestas de intervención.

\subsection{Algunas apreciaciones acerca de las estimaciones realizadas}

Debido a los numerosos análisis estadísticos realizados para estudiar adecuadamente las variables pertinentes del cuestionario ad hoc, se ha generado una cantidad considerable de información, por lo que no fue una tarea sencilla resumirla y reflexionar con ecuanimidad respecto de los resultados producidos referidos a la protección del suelo agrícola.

Por otra parte, los mismos han sido conseguidos a partir de las medidas recogidas, en atención a ello no serían generalizables puesto que responden a las características particulares de la muestra seleccionada para la presente investigación.

Así pues, del análisis de datos obtenidos se desprende que, de los 27 productores entrevistados, el $66.68 \%$, el $74.07 \%$ y el $81.48 \%$ realizan permanentemente rotación de cultivos, siembra directa con semilla transgénica y monitoreo de plagas respectivamente, por citar algunos ejemplos. Estas situaciones, si bien eran probables, no pueden ser generalizables; sin embargo, podría inferirse que: en gran número las explotaciones sojeras del Chaco realizan buenas prácticas agrícolas tendientes a la protección del recurso suelo.

El desarrollo que ha tenido éste trabajo en la etapa empírica -en lo atinente a la protección del suelo agrícola-, permite señalar que ha sido posible:

1) Explorar el comportamiento de las variables del cuestionario aplicado, a través del tratamiento de los datos obtenidos, lo que derivó en un gran número de indicadores descriptivos que resultan de utilidad para conocer, mediante información fehaciente, adecuada y actual, la caracterización que presentan las pequeñas empresas radicadas en el polo sojero del Oeste del 
Chaco, como provincia que no pertenece a la región pampeana.

2) Comprobar que los resultados alcanzados se encuentran en consonancia con los proporcionados por la literatura específica en la temática bajo análisis, ya que los resultados logrados comprobaron empíricamente una serie de presunciones teóricas que, implícita o explícitamente, se hallaban en cada una de los ítems evaluados. Como se comprenderá, la contrastación cuantitativa de éstos resultados, no fue posible por no haberse encontrado información con la cual poder compararlos.

\subsection{Planteos de investigaciones complementarias}

Con el objetivo de generar pautas objetivas para la evaluación de la sustentabilidad ambiental en explotaciones sojeras, se considera conveniente efectuar los siguientes planteos de investigaciones complementarias:

1) Ampliar el estudio a zonas de similares características sociales, económicas y culturales, para comparar si se repiten (o no) los patrones de comportamiento observados.

2) Proyectar la metodología a nuevos períodos de observación (siguiendo un diseño de tipo longitudinal), y de corresponder, la adecuación del instrumento de medición.

3) Replicar la captura de datos en otros cultivos, dado que muchas de las cuestiones señaladas y tratadas a lo largo de este trabajo son comunes.

\subsection{Propuestas de intervención}

A partir de los resultados que derivan de los análisis de datos formalizados oportunamente, se brindan a continuación propuestas de intervención, las que se pretende contribuyan con el desarrollo de las pequeñas empresas sojeras del Chaco.

I. Poner en práctica acciones destinadas a concientizar a los actores del sector acerca de la importancia que tiene el desarrollo de una explotación sustentable, particularmente en lo referido a la protección del suelo.

II. Profundizar la investigación sobre la temática bajo estudio, promoviendo la interdisciplinariedad y la participación de distintos sujetos vinculados a la actividad (ingenieros agrónomos, administradores, empresarios, entre otros).

III. Fomentar la construcción de bases de datos, por parte de organismos e instituciones tanto públicas como privadas, que permitan disponer de información a efectos de la realización de estudios sobre la materia objeto de interés.

En definitiva sería conveniente, a fin de fortalecer en la comunidad agrícola el desarrollo de prácticas sustentables, la puesta en marcha de esta serie de medidas de acción, absolutamente plausibles de implementar. 


\section{CONCLUSIONES}

Desde fines de los años 80 se han sucedido profundas modificaciones que rediseñaron la estructura productiva en el desarrollo de la actividad agrícola. Es por ello que en los resultados del Censo Agropecuario 2002 ya se observó que mientras la superficie utilizada se incrementaba, la cantidad de pequeñas empresas disminuía.

A su vez, el desarrollo del presente estudio lleva a sostener que la exploración de variables y análisis de los resultados, han permitido la consecución del objetivo del presente estudio, como paso necesario para validar la hipótesis sustantiva oportunamente formulada.

Asimismo, cabe resaltar que la totalidad de las variables que intervinieron en el cuestionario elaborado para esta investigación, resultaron útiles a fin de brindar una reseña sobre las particularidades de las explotaciones agrícolas sojeras chaqueñas. La información descriptiva al respecto, la cual se explicita a continuación, está basada en los resultados de los análisis cuantitativos implementados oportunamente.

- Datos personales: los productores agrícolas encuestados poseen una edad promedio de 48.70 años, con una desviación estándar de 12.91; predominando el sexo masculino (92.59\%) entre ellos. Por otra parte, cabe señalar, que sus explotaciones agrícolas se encuentran ubicadas en su mayor parte en el Departamento Chacabuco (51.72\%), cuya ciudad cabecera es Charata.

- Protección de los Recursos Naturales: los participantes del estudio demostraron conductas permanentes que propenden a la sustentabilidad, tales como: rotación de cultivos, implantación sobre rastrojos, adopción de siembra directa y semilla transgénica, como también el uso de agroquímicos y el monitoreo de plagas bajo asistencia técnica. Sin embargo, se debe fortalecer la alternancia de actividades económicas (agricultura-ganadería), la incorporación de fertilización balanceada y la realización periódica del estudio de suelo, tendientes al cuidado preventivo del mismo.

Finalmente, más allá de las limitaciones de recursos materiales y humanos que todo trabajo de investigación conlleva, se espera que este primer acercamiento, por nuestra parte, a la temática tratada constituya un aporte a la explotación agrícola sojera sustentable. Desde luego, también se anhela que este estudio represente una contribución razonable para la comunidad científica y profesional abocada a su asesoramiento e in-teresada en llevar a cabo futuras investigaciones en este área disciplinar.

\section{REFERENCIAS BIBLIOGRÁFICAS}

Asociación Argentina de Productores en Siembra Directa (Aapresid). (s.f.). Manual de buenas prácticas agrícolas e indicadores de gestión. Recuperado el 17 de julio de 2012 de www.aapresid.org.ar/ac/wp-content/uploads/sites/4/2013/o2/manual.pdf

Carbajal, M., Innocente, R., Jerez, S., de Castro, I. y García, P. (2011, octubre). Uso de agroquímicos y riesgo ecológico. La experiencia chaqueña. II Coloquio Nacional de Percepción y 
Gestión de Riesgo Agropecuario de la Universidad de Buenos Aires. Ciudad Autónoma de Buenos Aires, Buenos Aires, Argentina.

Casparri, M.T. y García Fronti, V. (2011). Los pequeños productores y los seguros agrícolas: encuesta exploratoria. En M. T. Casparri y A. S. Vilker (Eds.). Finanzas Agropecuarias en un contexto de incertidumbre (pp. 57-68). Buenos Aires, Argentina: Universidad de Buenos Aires.

Ministerio de Agricultura Ganadería y Pesca (2009). Instituto Nacional de Estadística y Censos (INDEC). Censo Nacional Agropecuario 2008. Recuperado el 14 de noviembre de 2012 de www.indec.gov.ar/censoagro2008/cna08_10_09.pdf

Fox, D. (1981). El proceso de investigación en educación. Pamplona, España: EUNSA.

Inciarte, R. (2004). Las buenas prácticas agrícolas. Organización de las Naciones Unidas para la agricultura y la alimentación. Oficina Regional de la FAO para América Latina y el Caribe. Recuperado el 14 de noviembre de 2012 de ftp.fao.org/docrep/fao/o10/AIo10s/AIo10soo.pdf

Ministerio de Agricultura Ganadería y Pesca. Red de Información Agropecuaria Nacional (RIAN). Instituto Nacional de Tecnología Agropecuaria (INTA). (2010). Zonificación RIAN Chaco y Formosa. Recuperado el 14 de diciembre de 2012 de inta.gob.ar/documentos/ rian-chaco-formosa-informe-mayo-2011/

Ministerio de Agricultura Ganadería y Pesca. Red de Información Agropecuaria Nacional (RIAN). Instituto Nacional de Tecnología Agropecuaria (INTA). (2011, mayo). Informe agropecuario para Chaco y Formosa, por Departamento. Recuperado el 17 de julio de 2012 de inta.gob.ar/documentos/rian-chaco-formosa-informe-mayo-2011/

Scheinkerman, E., Foti, M. P.y Román, M. E. (2007). Los pequeños productores de la República Argentina. Importancia en la producción agropecuaria y en el empleo en base al Censo Nacional Agropecuario 2002 (2a. Ed.). Serie estudios e investigaciones No 10. Buenos Aires, Argentina: Instituto Interamericano de Cooperación para la Agricultura y Ministerio de Agricultura, Ganadería y Pesca de la República Argentina.

Scheinkerman, E. (2009). Las explotaciones agropecuarias familiares de la República Argentina. Un análisis a partir de los datos del Censo Nacional 2002. Serie estudios e investigaciones $\mathrm{N}^{0}$ 23. Buenos Aires, Argentina: Instituto Interamericano de Cooperación para la Agricultura y Ministerio de Agricultura, Ganadería y Pesca de la República Argentina. 


\section{ANEXO}

\section{Anexo I: CUESTIONARIO DE SUSTENTABILIDAD EN PEASPC}

\section{Introducción}

El presente cuestionario ha sido diseñado como herramienta de recolección de datos empíricos (es decir datos basados en la experiencia) para un estudio que estamos realizando con el fin de conocer el grado de sustentabilidad en pequeñas explotaciones agrícolas sojeras de la Provincia del Chaco (PEASPC). Dichos emprendimientos empresariales se encuentran radicados en la zona oeste XVI-B con epicentro en la localidad de General Pinedo e integrada por los departamentos de General Belgrano, 9 de Julio, Chacabuco, 12 de Octubre y 2 de Abril.

En efecto, este instrumento está destinado a ser aplicado a sus productores, quienes han aceptado amablemente prestar colaboración con el fin de posibilitar el estudio indicado precedentemente.

Con ese objetivo, aparecen a continuación una serie de preguntas sobre distintos aspec-tos vinculados al funcionamiento de la explotación agrícola. Le pedimos que conteste con tranquilidad, ya que los datos de este cuestionario serán tratados de manera estrictamente confidencial y utilizados solamente con propósitos científicos por los responsables de esta investigación. Asimismo, cabe señalar que la contribución que pudiera brindarnos servirá para formular un diagnóstico y plantear alternativas que puedan serle de utilidad para el desarrollo de su futura actividad productiva, económica y financiera.

\section{Modo de responder}

A continuación se formularán distintas preguntas sobre la las decisiones adoptadas en las últimas tres campañas agrícolas y se seleccionará la opción que considere más cercana a su realidad.

En el primer grupo de preguntas se dispone para responder de una escala de 5 puntos, en la que se rodeará con un círculo el número que se entienda como la respuesta adecuada en cada uno de los enunciados que se presentan:

\begin{tabular}{|c|c|c|c|c|}
\hline 1 & 2 & 4 & 3 & 5 \\
\hline Desconoce & Nunca & Muchas veces & Pocas veces & Permanentemente \\
\hline
\end{tabular}

En el segundo grupo de preguntas se dispone de columnas donde se registrarán datos numéricos de las últimas tres campañas agrícolas. De no recordar todos los datos solicitados, se anotarán al menos los que correspondan a la campaña concluida en mayo de 2012. 
En el tercer grupo, en caso de corresponder, se encuentran cinco (5) ítems en los que se solicita que comente detalles de las decisiones adoptadas.

\section{I - Datos de identificación}

Fecha de realización:

Entrevistador:

Apellidos y Nombres del productor (opcional):

Localidad donde se ubica la explotación agrícola:

\section{II - Grado de sustentabilidad de la empresa agrícola sojera del Chaco}

\section{Primer grupo de preguntas}

En las últimas tres (3) campañas:

\begin{tabular}{|c|c|c|c|c|c|c|}
\hline 1 & $\begin{array}{l}\text { ¿Alternó la producción de soja con otros cultivos? } \\
\text { Ver si corresponde pregunta } N^{o} 26 \text {. }\end{array}$ & 1 & 2 & 3 & 4 & 5 \\
\hline 2 & $\begin{array}{l}\text { ¿Plantó soja sobre rastrojos de un cultivo invernal } \\
\text { inmediato anterior? }\end{array}$ & 1 & 2 & 3 & 4 & 5 \\
\hline 3 & ¿Alternó la producción de soja con ganadería? & 1 & 2 & 3 & 4 & 5 \\
\hline 4 & ¿Realizó solamente siembra directa? & 1 & 2 & 3 & 4 & 5 \\
\hline 5 & $\begin{array}{l}\text { ¿Plantó solamente semillas modificadas } \\
\text { genéticamente (transgénicas)? }\end{array}$ & 1 & 2 & 3 & 4 & 5 \\
\hline 6 & $\begin{array}{l}\text { ¿Realizó monitoreo a campo para identificar plagas } \\
\text { y malezas invasoras del cultivo? }\end{array}$ & 1 & 2 & 3 & 4 & 5 \\
\hline 7 & $\begin{array}{l}\text { ¿Mantuvo controlada (no eliminada) la población de } \\
\text { plagas y malezas invasoras del cultivo? }\end{array}$ & 1 & 2 & 3 & 4 & 5 \\
\hline 8 & $\begin{array}{l}\text { ¿Realizó una fertilización balanceada (manteniendo o } \\
\text { recuperando solo los componentes perdidos) al suelo? }\end{array}$ & 1 & 2 & 3 & 4 & 5 \\
\hline 9 & ¿Contrató asesoramiento profesional propio? & 1 & 2 & 3 & 4 & 5 \\
\hline 10 & $\begin{array}{l}\text { ¿Recibió asesoramiento profesional de terceros } \\
\text { (organismos oficiales, proveedores de insumos, etc.)? }\end{array}$ & 1 & 2 & 3 & 4 & 5 \\
\hline 11 & ¿Realizó algún tipo de estudio del suelo? & 1 & 2 & 3 & 4 & 5 \\
\hline 12 & $\begin{array}{l}\text { ¿Hace un balance de nutrientes luego de cada campaña? } \\
\text { Ver si corresponde pregunta } N^{o} 27 \text {. }\end{array}$ & 1 & 2 & 3 & 4 & 5 \\
\hline 13 & $\begin{array}{l}\text { ¿Contrató algún tipo de seguro agrícola? Ver si } \\
\text { corresponde pregunta } N^{o} 28 \text {. }\end{array}$ & 1 & 2 & 3 & 4 & 5 \\
\hline 14 & ¿Vendió su producción a acopiadores locales? & 1 & 2 & 3 & 4 & 5 \\
\hline 15 & $\begin{array}{l}\text { ¿Considera que ha obtenido un beneficio satisfactorio? } \\
\text { Ver si corresponde pregunta } N^{o} 29 \text { y } 30 .\end{array}$ & 1 & 2 & 3 & 4 & 5 \\
\hline
\end{tabular}




\begin{tabular}{|c|c|c|c|c|}
\hline \multicolumn{5}{|c|}{ Segundo grupo de preguntas } \\
\hline \multicolumn{2}{|c|}{ En las últimas tres (3) campañas: } & 2012 & $2012 / 2011$ & 2011 \\
\hline 16 & $\begin{array}{l}\text { ¿Cuál fue el rendimiento de soja? Esta pregunta se puede } \\
\text { responder indicando la producción obtenida por hectárea } \\
\text { cultivada ó por el total de has destinadas a la agricultura. }\end{array}$ & & & \\
\hline 17 & $\begin{array}{l}\text { ¿Cuál fue el precio recibido por la tonelada de soja? Esta } \\
\text { pregunta se puede responder indicando el precio obteni- } \\
\text { do por tonelada cosechada ó por el total de la producción. }\end{array}$ & & & \\
\hline 18 & ¿Cuántas hectáreas tuvo disponible para trabajar? & & & \\
\hline 19 & ¿Cuántas hectáreas destinó a la agricultura? & & & \\
\hline 20 & $\begin{array}{l}\text { ¿Cuántas hectáreas de la tierra que destinó a la agricultura } \\
\text { eran de su propiedad? Ver si corresponde pregunta } N^{o} 31\end{array}$ & & & \\
\hline 21 & $\begin{array}{l}\text { ¿Cuántos miembros de la familia entre } 18 \text { y } 65 \text { años vivie- } \\
\text { ron en la explotación? }\end{array}$ & & & \\
\hline 22 & $\begin{array}{l}\text { ¿Cuántos miembros de la familia entre } 18 \text { y } 65 \text { años tra- } \\
\text { bajaron en la explotación o en otro lado? }\end{array}$ & & & \\
\hline 23 & ¿Cuanto personal permanente contrató? & & & \\
\hline 24 & ¿Cuánto personal temporario contrató? & & & \\
\hline
\end{tabular}

\section{Tercer grupo de preguntas}

En las últimas tres (3) campañas:

25. ¿Con qué cultivos alternó la producción de soja?

26. ¿Cómo hace el balance de nutrientes luego de cada campaña?

27. ¿Qué cobertura contenía el seguro agrícola contratado? (Ejemplos: coberturas básicas: sequia, inundaciones, granizo o vientos fuertes. Coberturas adicionales: incendio, resiembra, heladas, lluvia en exceso, falta de agua, falta de piso y plagas o enfermedades) 
28. ¿Cuáles fueron los dos (2) factores físicos y/o económicos que considera tuvieron mayor importancia en el desempeño final de la explotación? (Ejemplos: eventos climáticos, políticas gubernamentales, acceso a tecnología, precios futuros, acceso y costo del financiamiento).

29. ¿Cómo resolvió los efectos negativos de los factores que afectaban la rentabilidad de la explotación?

30. La tierra destinada a la agricultura que no era de su propiedad, ¿̇como la obtuvo? ¿Cómo acordó el pago por el uso de la misma?

iMuchas gracias por su colaboración!

\section{CURRICULUM VITAE \\ Susana Gusinsky de Gelman}

Contadora Pública Nacional (1964), Universidad Nacional de Córdoba. Licenciada en Ciencias Económicas (1967) Universidad Nacional de Córdoba. Profesor Titular de la cátedra: Macroeconomía I (1973 y continúa a la fecha. Dedicación Exclusiva); Facultad de Ciencias Económicas - UNNE. Docente de grado y posgrado en temáticas del área Económica; Directora de tesinas de alumnos de grado. Directora y Co directora de proyectos de investigación (2003 a la fecha). Miembro de comisiones asesoras, de evaluación y de concurso. Secretaria de Investigación, Innovación y Desarrollo (mayo del 2010-2014). Investigador del Programa de Incentivos, categoría II (1999 a la fecha).

sgelman@eco.unne.edu.ar

\section{Rosa Cruz de Innocente}

Profesor en Ciencias Económicas (1987) y Especialista en Sindicatura Concursal (1998); Facultad de Ciencias Económicas de la Universidad Nacional del Nordeste. Profesor Titular de la cátedra: Contabilidad Básica (2013. Dedicación Exclusiva) y Profesora adjunta con dedicación 
simple de la cátedra: Estados Contables; Facultad de Ciencias Económicas - UNNE. Docente de grado y posgrado en temáticas del área Contable; Co directora de proyectos de investigación (2011 a la fecha). Miembro de comisiones asesoras, de evaluación y de concurso. Secretaria de Asuntos Estudiantiles y Secretaria de Extensión y Ejercicio Profesional de la Facultad de Ciencias Económicas de la Universidad Nacional del Nordeste (mayo del 2010-2014). Investigador del Programa de Incentivos, categoría IV (2010 a la fecha).

\section{rcruz@eco.unne.edu.ar}

\section{Susana Rosa Jerez}

Contador Público (1989); Facultades de Ciencias Económicas. Universidad Nacional del Nordeste. Especialista en Contabilidad Superior y Auditoria (2007) UNNE. Profesor adjunto por concurso, a cargo de la cátedra: Contabilidad para Administradores y Economistas de la Facultad de Ciencias Económicas de la Universidad Nacional del Nordeste (2011 a la fecha) y auxiliar docente de la cátedra Estados Contables, (2004-2016). Integrante del equipo de investigación del proyecto titulado "Figuras asociativas en la producción de soja en el Chaco. Grado de desarrollo de su información contable" 2013-2016, que se lleva a cabo en el ámbito de la Facultad de Ciencias Económicas de la Universidad Nacional del Nordeste. Fiscal del Tribunal de Cuentas de la Provincia del Chaco (1997 a la fecha).

sjerez@eco.unne.edu.ar

\section{Margarita Carlota Carbajal}

Contador Público Nacional (1971); Profesora en Ciencias Económicas (2004). Facultades de Ciencias Económicas. Universidad Nacional del Nordeste. Especialista en Tributación (2000). Facultad de Ciencias Económicas. Universidad Nacional del Nordeste. Máster en Derecho Tributario, Universitat de Barcelona (España), 2002. Profesor adjunto a cargo de la cátedra: Régimen Tributario; Profesor adjunto de la cátedra: Impuestos II. Consejera Directiva Titular por el claustro de profesores adjuntos, Consejo Directivo de la Facultad de Ciencias Económicas UNNE (2011 a la fecha). Jefe Titular de la División Capacitación de la Administración Federal de Ingresos Públicos. Dirección General Impositiva. Región Resistencia (1999 a la fecha).

margacarbajal@hotmail.com

\section{Idalia Gabriela de Castro}

Especialista en Contabilidad Superior y Auditoria (2007), Facultades de Ciencias Económicas. Universidad Nacional del Nordeste. Docente de la Facultad de Ciencias Económicas de la Universidad Nacional del Nordeste en la cátedra Contabilidad Básica, como profesor adjunto con dedicación exclusiva (2003-2014) y en la cátedra Estados Contables, como auxiliar docente con dedicación simple (2002-2014). Es integrante del equipo de investigación de los proyectos titulados "Modelización estadística del rendimiento académico en estudiantes de Ciencias Económicas” 2011-2014 y "Figuras asociativas en la producción de soja en el Chaco. Grado de desarrollo 
SUSANA G. DE GELMAN - ROSA CRUZ DE INNOCENTE - SUSANA ROSA JEREZ - MARGARITA CARLOTA CARBAJAL - IDALIA DE CASTRO $>106$ NIVEL DE SUSTENTABILIDAD EN PEQUEÑAS EXPLOTACIONES SOJERAS DE LA PROVINCIA DEL CHACO - SUELO AGRÍCOLAPags. $82-107$

de su información contable" 2013-2016; ambos se llevan a cabo en el ámbito de la Facultad de Ciencias Económicas de la Universidad Nacional del Nordeste.

idecastro@eco.unne.edu.ar 